\title{
Influence of Surface Topography on the Surface Durability of Steam Oxidised Sintered Iron
}

\author{
José Daniel Biasoli de Mello* \\ Laboratório de Tribologia e Materiais, Universidade Federal de Uberlândia, \\ Bloco 1R, 38400-902 Campus Santa Monica, Uberlândia - MG, Brazil
}

Received: November 23, 2003; Revised: March 13, 2005

\begin{abstract}
Durability of surfaces has been reported as the main factor affecting tribological behavior of steam oxidised sintered iron. The presence of surface pores and their negative influence on load bearing capacity, suggest that surface topography might play an important role on the durability of the oxide layer. In this paper, the influence of compaction pressure and powder grade on surface topography, and as a consequence, its effect on the tribological behavior of steam oxidised sintered iron has been analysed. Specimens prepared from atomised iron powders with different sizes were compacted using 4 different pressures, sintered, and then subjected to steam treatment. Tribological characterisation was carried out in a reciprocating sliding wear test. Although the processing parameters affected the surface topography to a considerable extent, the main influence may be attributed to powder grade. A strong influence of surface topography on the durability distance, evaluated in terms of the evolution of contact resistance with total sliding distance, has been highlighted. Surfaces which were smoother and had high load-carrying capacity were always associated with a higher durability distance.
\end{abstract}

Keywords: surface durability, surface topography, steam oxidation, sintered iron

\section{Introduction}

Sintered iron-based materials have been used for load bearing parts in sliding contact such as plain bearings, gears and brake linings, because of their good mechanical properties and low cost when produced in high volume ${ }^{1,2}$.

In addition to a reduction in strength and, as a consequence, in load bearing capacity, the presence of pores might influence in a number of ways the wear mechanisms acting on the surfaces of parts produced by powder metallurgy $y^{3-8}$.

In the sintering industry, steam oxidation is a frequently used secondary operation, with fairly low production costs which require little attention during operation ${ }^{9}$. While initially used with the purpose of sealing the network of interconnected pores and thus making the component impervious to liquid and gases, steam treatment is claimed to improve other properties of sintered iron.

The oxide formed on the surface and in the interconnected pores is reported to provide an increase in hardness and in mechanical properties as a whole, and wear resistance in particular, a decrease in friction coefficient and an increase in corrosion resistance in moderately aggressive environments ${ }^{9-12}$. Oxidation conditions are relatively well established which yield sintered iron products with the required properties ${ }^{9-15}$.

The effect of compaction pressure and powder grade on the microstructure and hardness of steam oxidised sintered iron has been extensively described in a recent paper by De Mello and co-authors ${ }^{16}$. A clear influence of the processing parameters on porosity and oxide content was highlighted. Low porosity was always associated with high compaction pressure and larger powder size. Pore size was affected in the same way by compaction pressure, even though the powder size had the opposite effect. Changes in compaction pressure and powder size had no significant effect on pore shape. The mean free path between pores increased with the powder grade (particle size) and reached a maximum as the compaction pressure increased. The value of this maximum increased as the powder grade decreased.
As expected, for a constant compaction pressure, the mean free path between pores decreased almost linearly with porosity. The amount of oxide formed inside the interconnected pores decreased as the compaction pressure and powder size increased. Further, the oxide content was strongly dependent on porosity. Although the steam treatment had a strong influence on macro hardness, it was not affected by the presence of surface oxide. The oxide inside the interconnected pores, by contrast, had a considerable effect on hardness. Decreasing powder size always lead to high hardness. Samples produced with powders of smaller size showed a continuous decrease in hardness as the compaction pressure increased, although for the large sized powder there was a slight increase, to a constant value, in ultimate hardness. For the intermediate powder size a maximum hardness was obtained as the compaction pressure increased. No general correlation was found between processing parameters and micro-hardness of the oxide layer itself or the ferrous matrix. Nevertheless, the oxide layer produced considerable surface hardening.

There have been few tribological studies of steam treated components ${ }^{17-18}$. Important contributions include the recent work of Straffelini and Molinari ${ }^{2,11,19-21}$. They studied the effect of sliding speed on the dry sliding behavior of sintered and steam oxidised iron and iron based alloys. They found that the wear rate was low and independent of the chemical composition of the specimens as long as the oxide layer, produced by the steam oxidation, is able to perform a lubricating action between the sliding surfaces.

These authors have analysed the effect of load on the tribological behavior of steam treated iron based alloys and introduced the concept of durability distance ${ }^{20}$. The concept of surface durability was related to the wear resistance of the oxide layer on the external surface and reported to be the main factor affecting tribological behavior $^{20-21}$. If the layer remains undamaged on the surface, wear resistance is higher, and greater than that provided by conventional heat treatment or case hardening ${ }^{21}$. 
As pointed out by Molinari and Straffelini ${ }^{20}$ the presence or absence of an undamaged layer of oxide on the surface was of paramount importance to the tribological behavior of iron-based steam oxidised alloys.

De Mello and Hutchings ${ }^{22}$ recently reported the influence of compaction pressure and powder grade on the surface durability of steam treated sintered iron. The tribological characterisation was carried out in a reciprocating ball-on-flat wear test. A strong influence of the processing parameters on durability distance, evaluated by means of changes in contact resistance was identified. The most durable layers were obtained by the combination of high compaction pressure with fine powder grade. For the same powder grade, an increase in compaction pressure lead to a large increase in durability distance. On the other hand, for the same compaction pressure, an increase in powder size gave rise to a marked reduction in durability. By analysing the wear mechanisms they concluded that the removal of the surface oxide layer resulted from its transfer to the counter-body and the localised formation of small particles of debris. Some of the particles were trapped by the pores and some were fragmented and deformed, starting the process of pore closure. They showed that pore closure played a very important role in surface durability. In the low durability samples, some pores remained open at the surface and acted as preferential sites for the generation and trapping of wear debris, enhancing the process of oxide and metallic debris generation and thus, decrease in surface durability.

The surface of steam oxidised sintered iron parts is affected by the level of porosity and may be quite irregular. The presence of surface pores and their negative influence on the load bearing capacity and surface durability might suggest that surface topography also plays an important role on the durability of the oxide layer.

In this paper, the influence of compaction pressure and powder grade on surface topography, and as a consequence, its effect on the tribological behavior of steam oxidised sintered iron, have been studied.

\section{Experimental Procedure}

Specimens were produced from Ancor Steel 1000B Höganäs atomised iron powder. The powder was sieved into four different size fractions (particle diameter $\phi<65 \mu \mathrm{m}, 65-90 \mu \mathrm{m}$; 90-125 $\mu \mathrm{m}$ and $>125 \mu \mathrm{m}$ ) leading to four different powder grades (denoted 1 to 4 respectively). After mixing with $0.8 \mathrm{wt}$. (\%) zinc stearate as a lubricant, the powders were compacted at four different compaction pressures $(300,400,500,600 \mathrm{MPa})$ with a double action automatic press, in such a way that the surfaces to be tested were in contact with the moving punch.

The samples were designated as Axy where $x$ is compaction pressure divided by 100 and $y$ is the powder grade. For example: A34 denotes $300 \mathrm{MPa}, \phi>125 \mu \mathrm{m}$; A61 denotes $600 \mathrm{MPa}, \phi<65 \mu \mathrm{m}$ ). The resulting compacts, $50 \mathrm{~mm}$ in length by $10 \mathrm{~mm}$ width with height depending on compaction pressure and powder grade, were sintered in an industrial furnace in a mildly reducing atmosphere $\left(\mathrm{N}_{2}+10 \% \mathrm{H}_{2}\right)$ at $1120{ }^{\circ} \mathrm{C}$ for $25-30$ minutes. After cooling to room temperature the samples were steam treated. The steam treatment was carried out at $540{ }^{\circ} \mathrm{C}$ for 2 hours in a continuous industrial furnace.

The surface topography measurements were carried out with a Wyko 3D non-contact optical profilometer in the vertical scanning mode. The surface topography was characterised in terms of roughness $\left(R_{q}\right)$, wavelength of the irregularities $\left(\lambda_{q}\right)$ and bearing area associated parameters $\left(R_{k}, R_{p k}\right.$ and $\left.R_{v k}\right)$.

The roughness was evaluated from:

$$
R_{q}=\sqrt{\frac{1}{M \cdot N}} \sum_{k=1}^{M} \sum_{j=1}^{N} Z_{j k}^{2}
$$

where $R_{q}$ is the three-dimensional root mean square (r.m.s.) roughness, $\mathrm{Z}$ is the height of the irregularities on the surface, $\mathrm{M}$ and $\mathrm{N}$ are the number of data points in each direction of the array.

Additionally, the wavelength of the irregularities $(\lambda)$ was calculated from:

$$
\lambda_{q}=2 . \pi \cdot \frac{R_{q}}{\Delta_{q}}
$$

where $\lambda_{q}$ is the r.m.s. average wavelength, $R_{q}$ is the three-dimensional r.m.s. roughness and $\Delta_{q}$ is the r.m.s. average slope of the profile.

The average slope is given by the following equations:

$$
\Delta_{q}=\sqrt{\frac{1}{N}} \sum_{i=1}^{N}\left(\Delta_{i}\right)^{2}
$$

where $\mathrm{N}$ is the number of sampling points in the evaluation length and $\Delta_{i}$ is

$$
\Delta_{i}=\frac{1}{60 \cdot d_{o}}\left(Z_{i+3}-9 Z_{i+2}+45 Z_{i+1}-45 Z_{i-1}+9 Z_{i-2}-Z_{i-3}\right)
$$

where $\mathrm{d}_{\mathrm{o}}$ is the sampling interval between the profile points.

The bearing area associated parameters $\left(R_{k}, R_{p k}\right.$ and $\left.R_{v k}\right)$ are illustrated in Figure 1.

The reduced peak height $\left(R_{p k}\right)$ relates to the top portion of the surface which will be worn away in the running-in period of a porous iron bearing. It can be combined with other parameters derived from the bearing ratio to provide criteria for the ability of a surface to support a load or resist wear. The core roughness depth $\left(R_{k}\right)$, represents the working part of a surface and will carry the load after the initial running-in period. The reduced valley depth $\left(R_{v k}\right)$ is represented by the lowest part of the surface and has the function of retaining the lubricant. Their definitions and calculation procedures are described in detail in the literature ${ }^{23}$. The measurements were made in high resolution mode corresponding to an area of 1.0 by $1.3 \mathrm{~mm}$. The results represent an average of at least four measurements for every sample.

The tribological characterisation was carried out in a reciprocating wear test, previously described ${ }^{22}$. The friction force (via load cell) and contact resistance between the sliding surfaces were continuously monitored and logged, through a 12 bit A/D card, on to a microcomputer. The coefficient of friction was calculated from the recorded tangential force and the normal load applied to the specimen. The durability of the oxide layer was determined by penetration of the surface oxide and was defined as the sliding distance at which the value of the contact resistance was $<50 \Omega$ for at least 10 seconds $^{22}$.

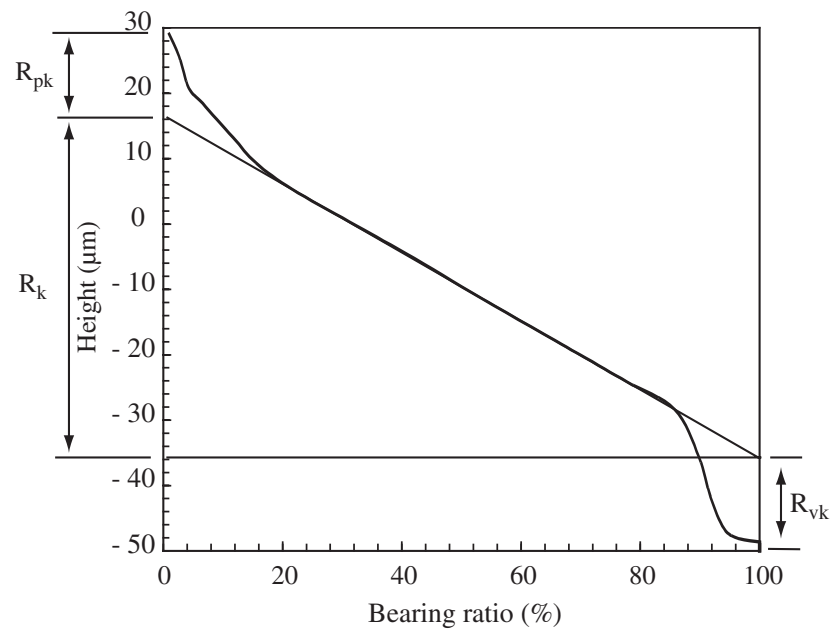

Figure 1. Bearing ratio curve showing $R_{k}, R_{p k}$ and $R_{v k}^{23}$. 
To study the wear mechanisms and oxide layer durability, the specimen surfaces were examined in a Jeol 820 scanning electron microscope at a tilt angle of $35^{\circ}$ and by vertical scanning optical interferometry (Wyko 3D).

The tests were performed at a normal load of $12.30 \mathrm{~N}$. The load was optimised to give good discrimination between specimens with test times which were not unduly long ${ }^{22}$. The test duration was thus fixed at either 1200 seconds or 1800 seconds, depending on the durability of the oxide layer.

The counter-body was a $12.7 \mathrm{~mm}$ diameter AISI 52100 hard steel ball. The ball surface was used in the as-received condition and a new surface region was used for each test. Before the tests, balls and samples were thoroughly cleaned with ethanol followed by acetone in an ultrasonic cleaner, and then dried in hot air.

The test conditions are listed in Table 1 . The results shown represent an average of at least five tests under identical experimental conditions.

Table 1. Tribological test conditions.

\begin{tabular}{lc}
\hline Normal load $(\mathrm{N})$ & 12.3 \\
Initial Hertz contact pressure $(\mathrm{MPa})$ & 558 \\
Stroke $(\mathrm{mm})$ & 12 \\
Frequency $(\mathrm{Hz})$ & 1.85 \\
Number of cycles & 2200,3400 \\
Temperature $\left({ }^{\circ} \mathrm{C}\right)$ & $23 \pm 2$ \\
Medium & air \\
Lubricant & none \\
\hline
\end{tabular}

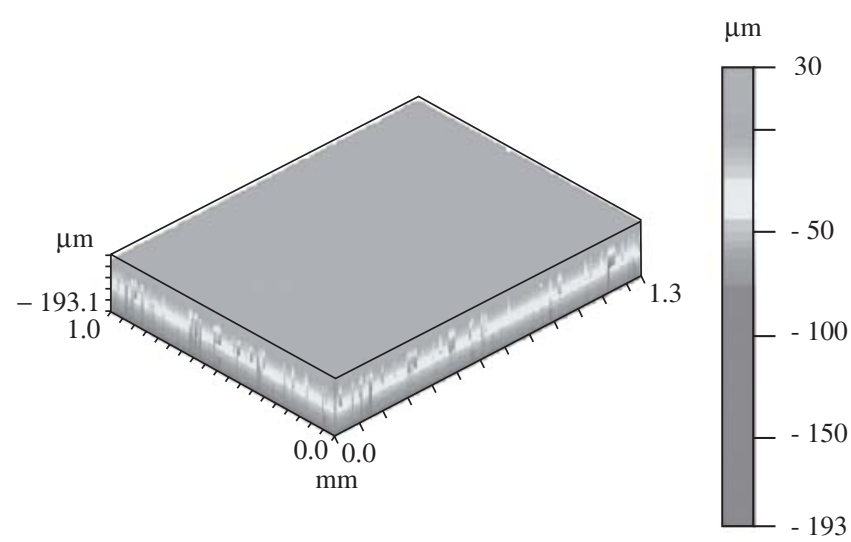

(a)

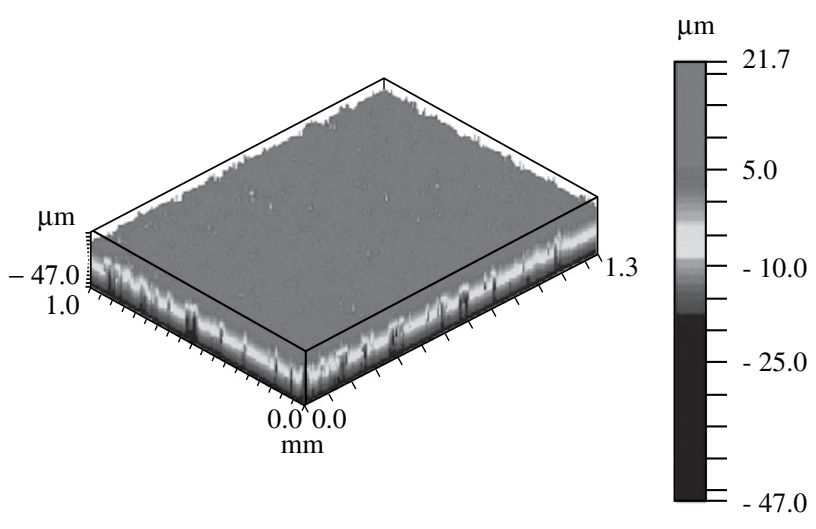

(c)

\section{Results and Discussion}

\subsection{Surface topography}

As a consequence of the processing parameters, which led to a high level of porosity, the surfaces of the samples were quite irregular. Figure 2 shows some typical aspects of the surfaces at magnifications corresponding to a macro-scale (by vertical scanning interferometry) as well as a micro-scale (by scanning electron microscopy). The presence of pores, although partially blocked by the iron oxide, considerably affects the surface topography. SEM shows, in addition, that the oxide layer covers the surface uniformly, including within the pores.

The variation of the root mean square (r.m.s.) roughness $\left(R_{q}\right)$ with the processing parameters is presented in Figure 3. As shown by the micrographs, the surfaces are quite rough. Although the roughness decreases slightly with increasing compaction pressure, powder grade has a significant influence. The roughness increases to a relatively constant value as the powder size increases. Even the smallest compaction pressure (300 MPa) is significantly higher than the yield stress of pure iron (180-220 MPa). During the compaction process, the surfaces of the powder particles in contact with the moving punch are flattened by compressive deformation. The surface is therefore composed of flattened regions originating from the deformed iron particles, and inter-particle valleys, corresponding to the pores.

Increase in compaction pressure, despite its strong effect in reducing porosity ${ }^{16}$, does not significantly affect the plateaux and thus the roughness. Increase in powder size, apart from affecting surface

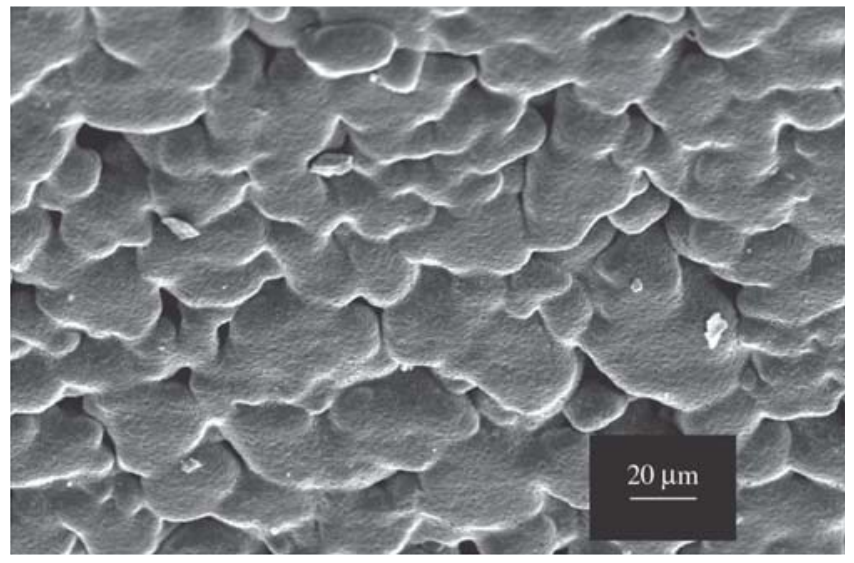

(b)

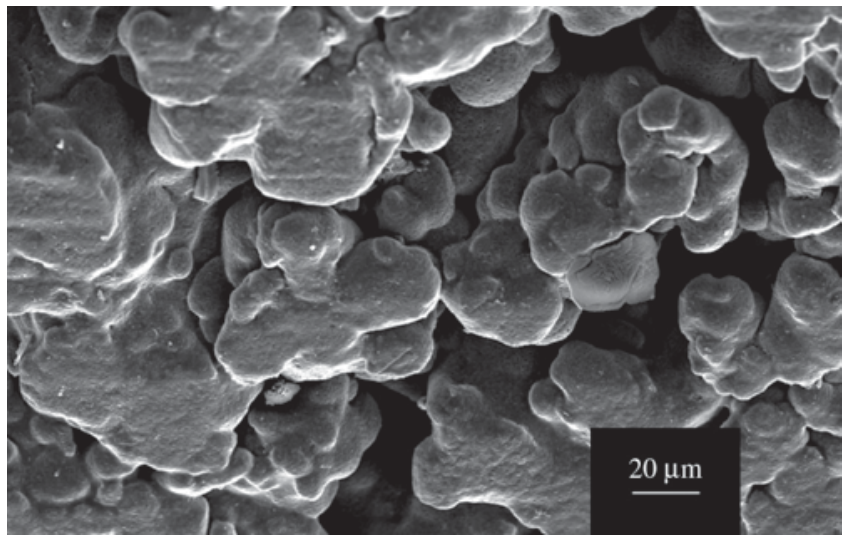

(d)

Figure 2. Typical surfaces. Sample A51 (500 MPa, $\phi<65 \mu \mathrm{m})$ a) VSI; b) SEM. Sample A34 (300 MPa, $\phi<125 \mu \mathrm{m})$; c) VSI; d) SEM. 


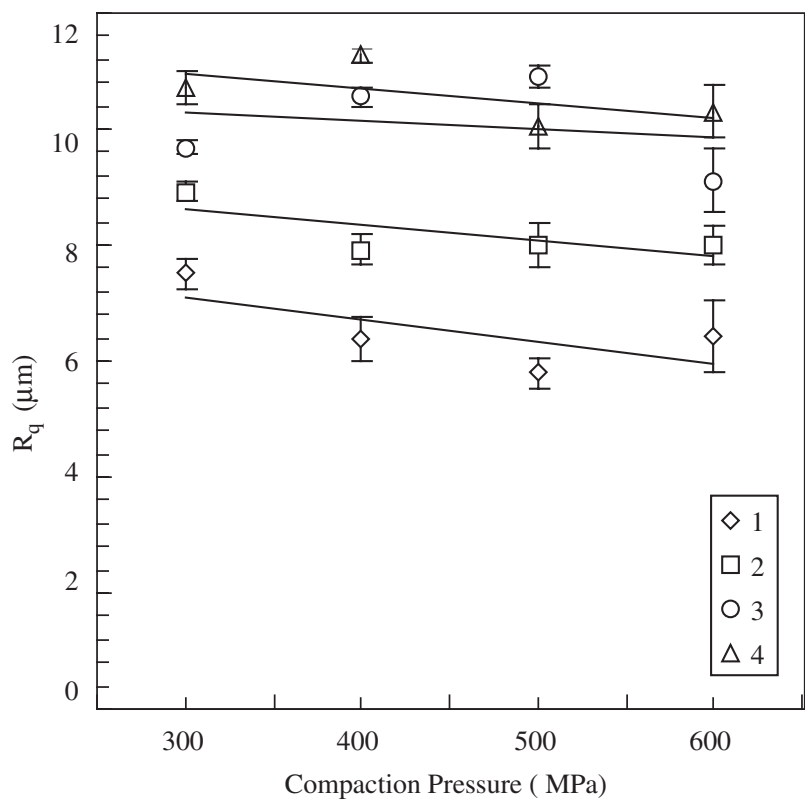

(a)

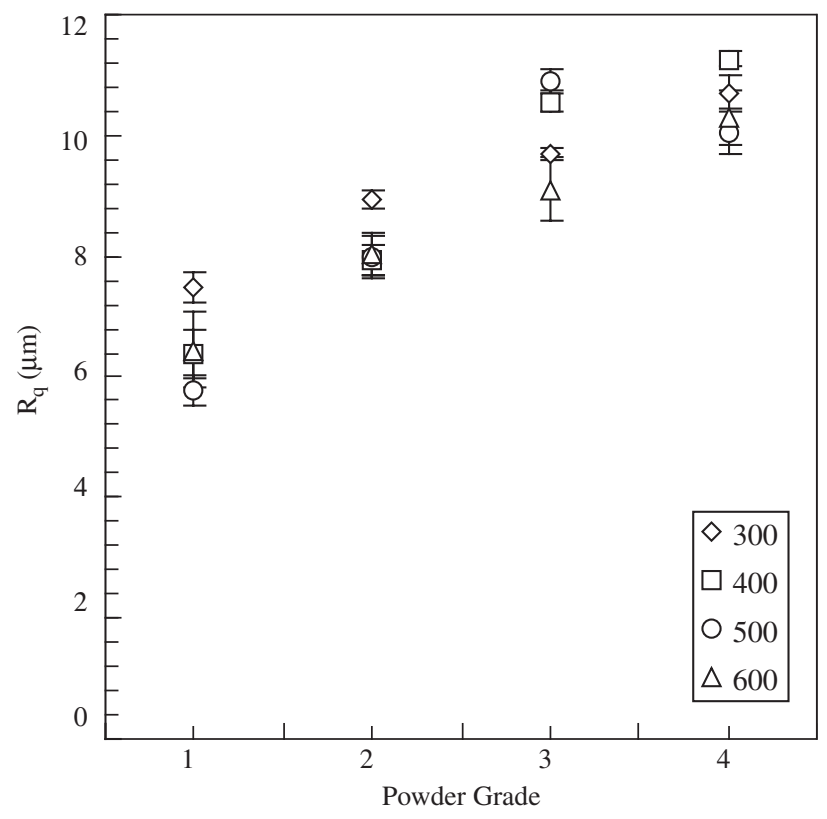

(b)

Figure 3. Surface roughness as a function of: a) compaction pressure; b) powder grade.

topography as a whole, produces deeper pores and, as a consequence, increased roughness.

For complete characterisation of the surface texture it is necessary to describe the way in which the hills, plateaux and valleys are distributed across the surface. Among the possible parameters that could serve as a 'horizontal descriptor', the r.m.s average wavelength $\left(\lambda_{q}\right)$, which represents the openness or closeness of the texture ${ }^{23}$, is perhaps appropriate for the study of wear of sintered materials. It is a measure of the distance between local peaks and valleys, taking into account their relative amplitudes and individual spatial frequencies; $\lambda_{q}$ is related to the power spectrum and represents a weighted mean of the Fourier spectrum. The correlation between the processing parameters and $\lambda_{q}$ as well as the reduced peak height $\left(R_{p k}\right)$, obeys the same general trends as seen for roughness (Figure 4 ).

Again, the powder grade is the main factor affecting surface topography. Increasing the compaction pressure produces a slight decrease in both the r.m.s. average wavelength $\left(\lambda_{q}\right)$, and the peak height $\left(R_{p k}\right)$

The reduced peak height $\left(R_{p k}\right)$ relates to the top portion of the surface which will be worn away for a porous iron bearing in the running-in period. It can be combined with other parameters derived from the bearing ratio to provide criteria for the ability of a surface to support a load or resist wear. The core roughness depth $\left(R_{k}\right)$, relates to the working part of a surface, which will carry the load after the initial running-in period. These parameters have been reported to correlate the life and performance of parts in sliding wear ${ }^{23}$. Increasing the powder size leads to an increase in $R_{k}$ to a constant value (Figure 5-b). The influence of compaction pressure on $R_{k}$ is shown in Figure 5-a.

In general, $R_{k}$ decreases as the compaction pressure increases. A critical level is evident, leading to two different magnitudes of core roughness depth.

\subsection{Tribological behavior}

The durability distance of the oxide layer varied strongly with the r.m.s. roughness, as shown in Figure 6. The results are spread between upper and lower bounds. In a general manner, lower values of r.m.s. roughness are associated with higher durability distance. Increasing the r.m.s. roughness leads to a rapid decrease in durability distance, which then becomes nearly independent of r.m.s. roughness. As stated before, the r.m.s. roughness is a measure of the amplitude of the irregularities of the surface and gives no information as to their shape. The durability of the surface oxide layer is strongly dependent on pore closure ${ }^{22}$ which in turn depends on the formation of small particles of debris. As the quantity, size and closeness of the pores can play a role in the pore closure process, it seems that the r.m.s average wavelength, a hybrid parameter determined from both amplitude and spacing information, should be a more useful parameter to establish the effect of surface topography on surface durability.

Figure 7 shows the relationship between the r.m.s. average wavelength and the durability distance.

Again, a spread in the results is evident. The variation of the durability distance with the r.m.s. average wavelength shows the same general behavior as for the r.m.s. roughness, indicating that the closeness of the pores does not have an important influence on the durability distance.

The effect of bearing ratio associated parameters on the durability distance is presented in Figure 8. Increase in these parameters correlates with rapid degradation of the surface oxide layer during sliding.

On the other hand, the surface durability, represented by the durability distance, is strongly associated with the removal of the surface oxide layer. The removal of this layer is gradual and results from local detachment of fine debris. Some of the debris is trapped by the pores and some are fragmented and deformed, due to the high compressive pressures acting at the contact, starting the process of pore closure. As a consequence, the surface of the wear scar becomes progressively smoother ${ }^{22}$. It is reasonable to suppose that the top portion of the surface can act as preferential sites for the generation of debris, which combined with pore closure, can explain the strong effect of surface topography on the surface durability.

It is interesting to note that although the analysis of the durability distance in terms of microstructure and processing parameters has 


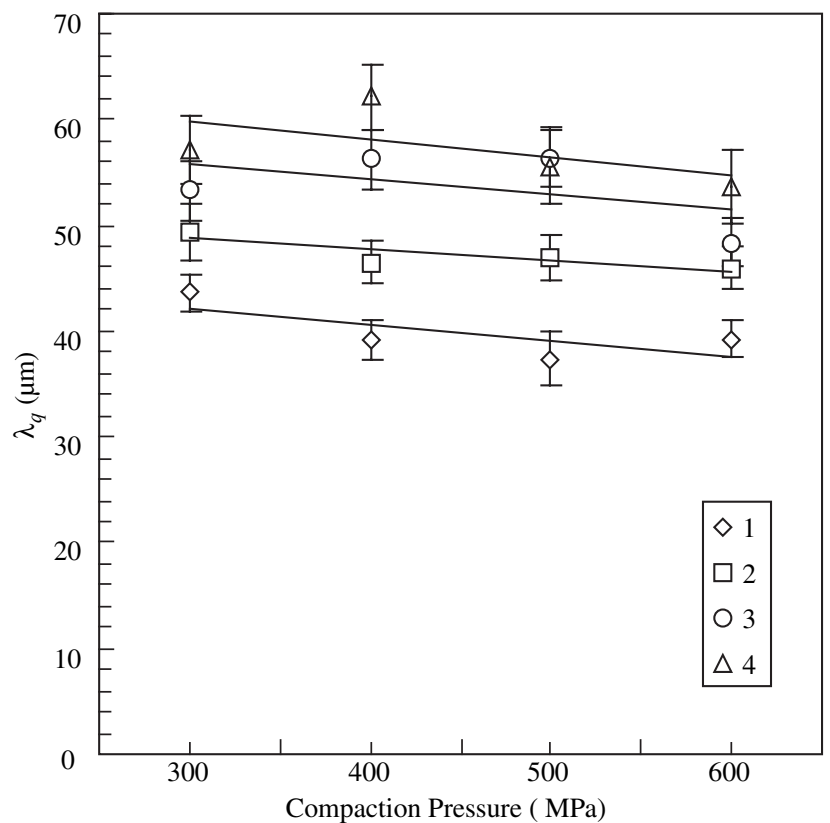

(a)

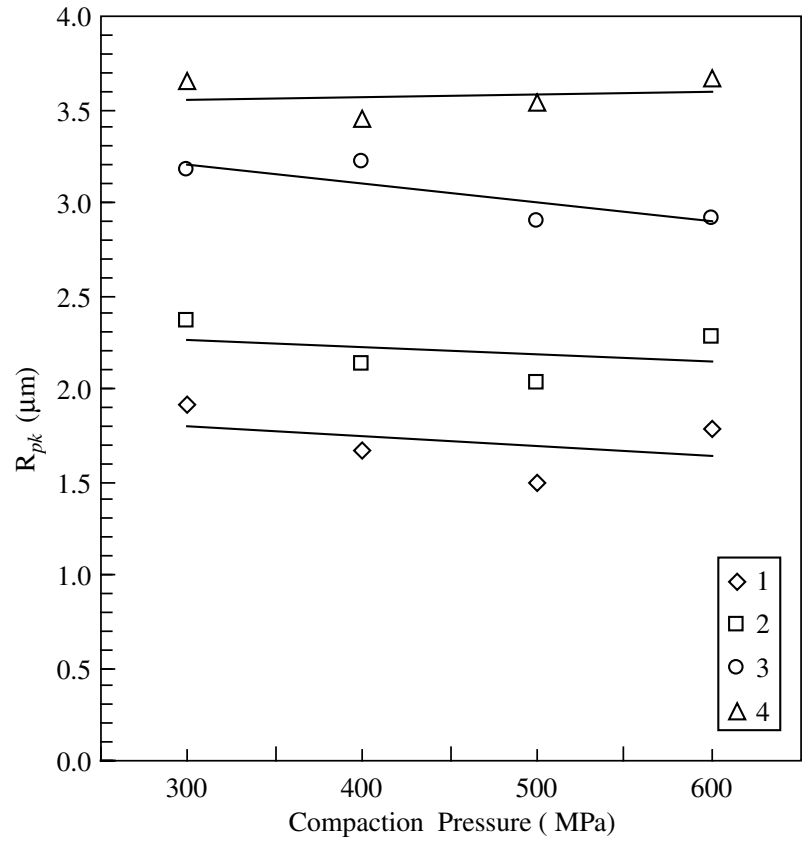

(c)

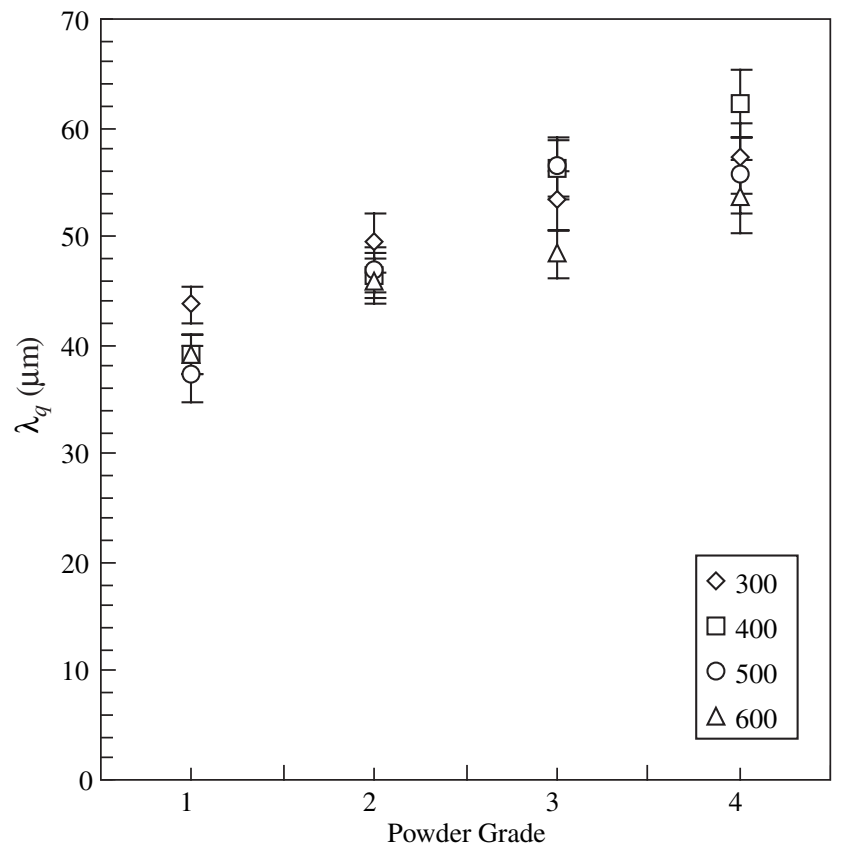

(b)

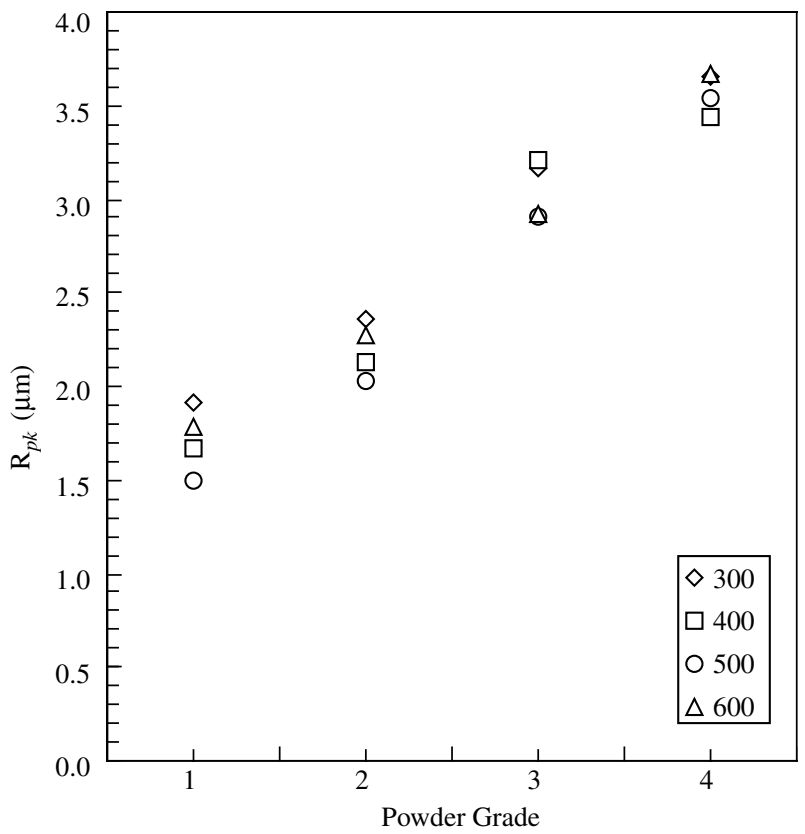

(d)

Figure 4. Influence on r.m.s. average wavelength of: a) compaction pressure; b) powder grade and on reduced peak height of; c) compaction pressure; d) powder grade.

shown that the most durable layers were obtained by the association of high compaction pressure with a small powder grade, the present analysis indicates that the use of small powders is more effective in controlling the topographical features. In fact, the present results clearly show that reducing powder grade always leads to lower values values of r.m.s. roughness, r.m.s. average wavelength, reduced peak height and core roughness depth, which in turn induce higher surface durability. Further, the evolution of these parameters with powder grade is nearly linear, suggesting that the powder grade should be a more convenient variable from an industrial point of view.

\section{Conclusions}

1. Although the processing parameters significantly affected the surface topography, the main influence was from powder grade. The r.m.s. roughness decreased slightly with increasing compaction pressure, and increased to a relatively constant value as the powder size increased. The correlation between the processing parameters and r.m.s average wavelength, as well as the reduced peak height, obeyed the same general trend. Increasing the powder size led to an increase in core roughness depth to a constant value. In general, core roughness depth decreased as the compaction pressure increased. 


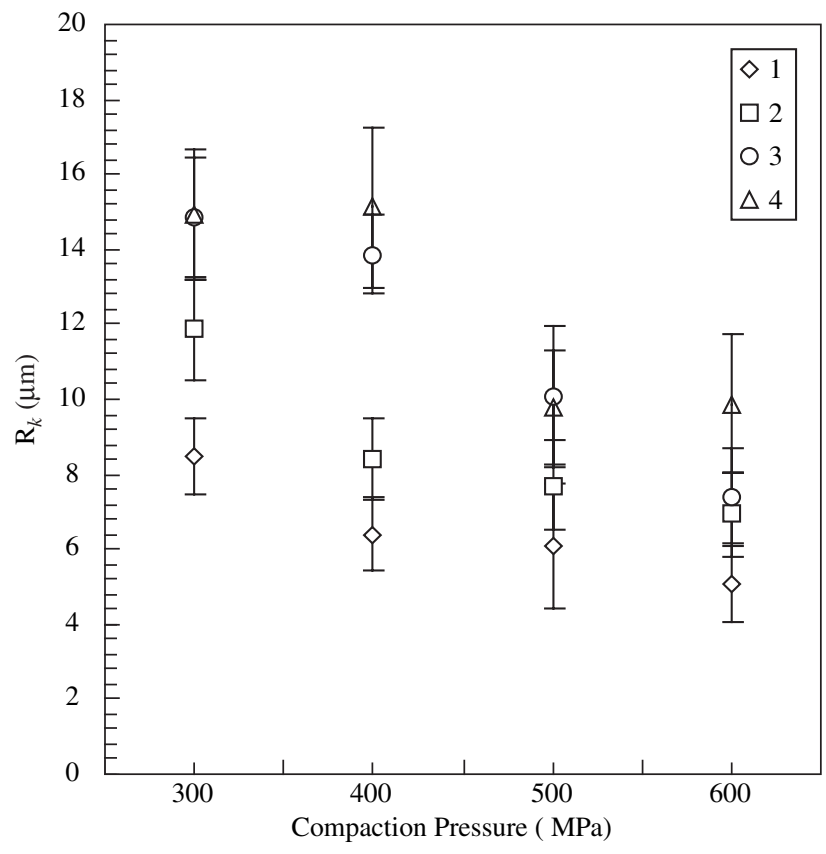

(a)

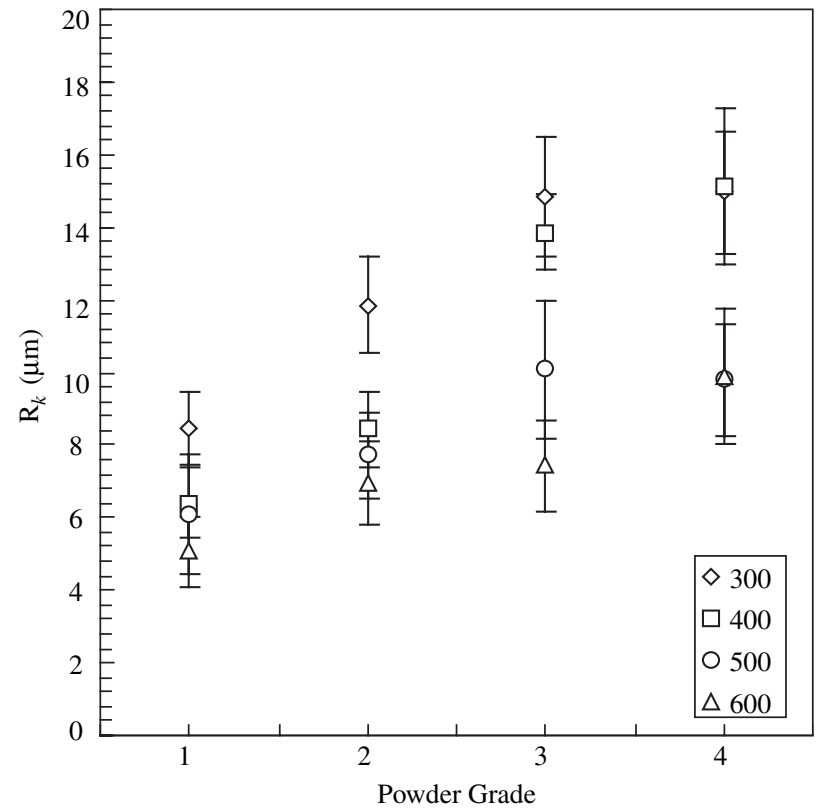

(b)

Figure 5. Variation of the reduced peak height with: a) compaction pressure; b) powder grade.

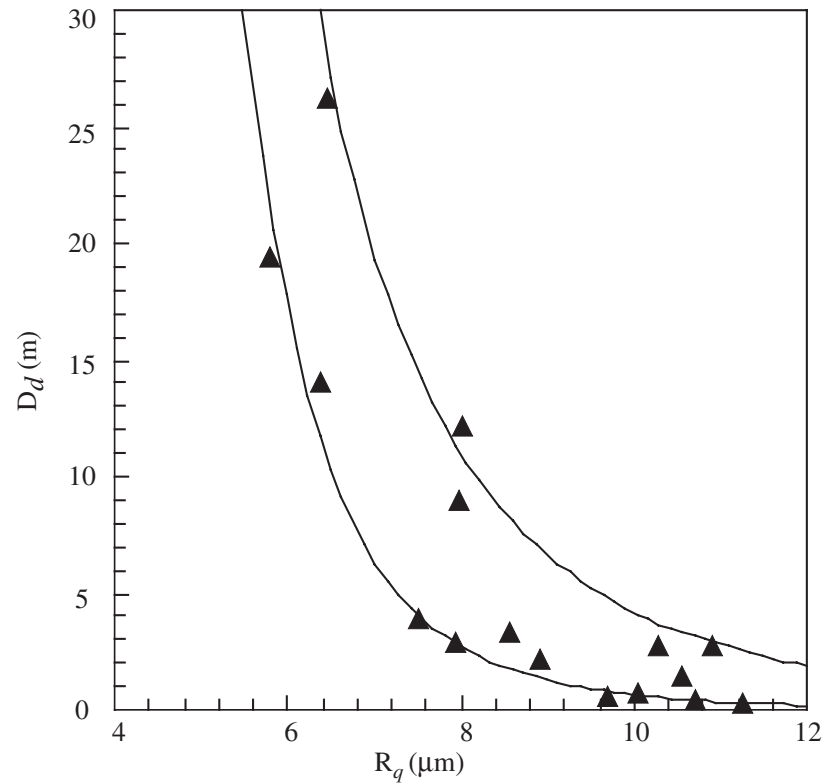

Figure 6. Influence of root mean square r.m.s. roughness on the durability distance.

2. The durability distance of the oxide layer was determined in a reciprocating dry sliding test against a hard steel ball and was strongly influenced by surface topography. Smoother surfaces with a high load-carrying capacity were always associated with greater durability distance. Increasing the r.m.s. roughness led to a rapid decrease in durability distance, which then became nearly independent of r.m.s. roughness. The variation of the durability distance with r.m.s. average wavelength showed

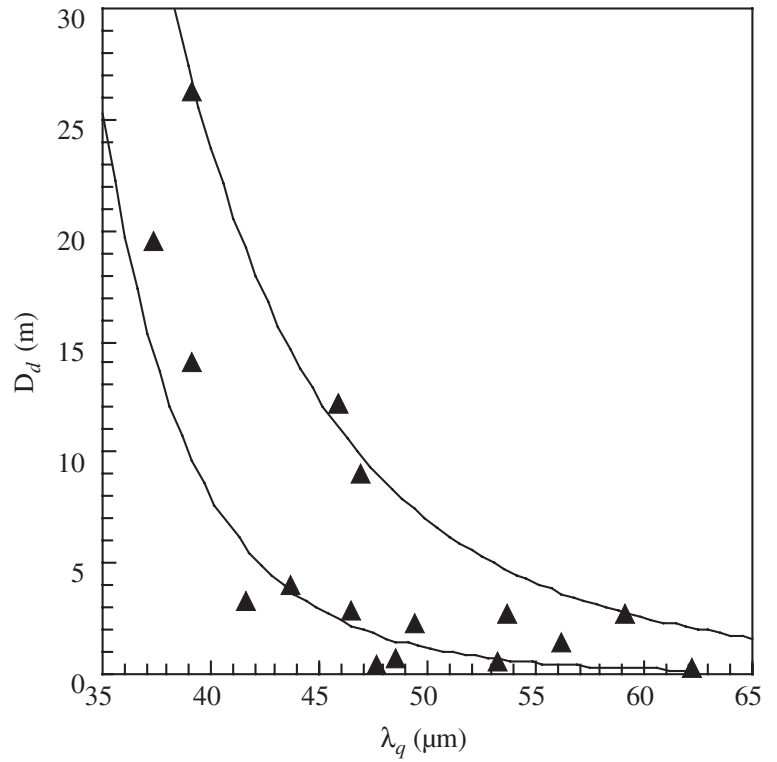

Figure 7. Relationship between r.m.s average wavelength and durability distance.

the same general behavior. Increasing bearing ratio associated parameters led to rapid degradation of the surface oxide layer.

\section{Acknowledgments}

The authors would like to thank the CNPq, CAPES and FAPEMIG, Brazil for their financial support. The work was performed at the University of Cambridge, UK. 


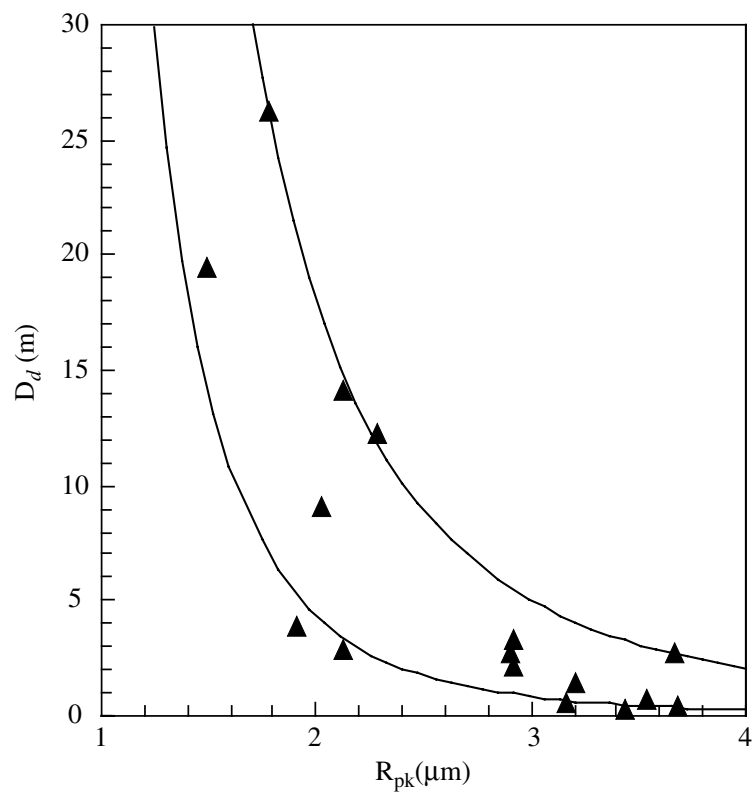

(a)

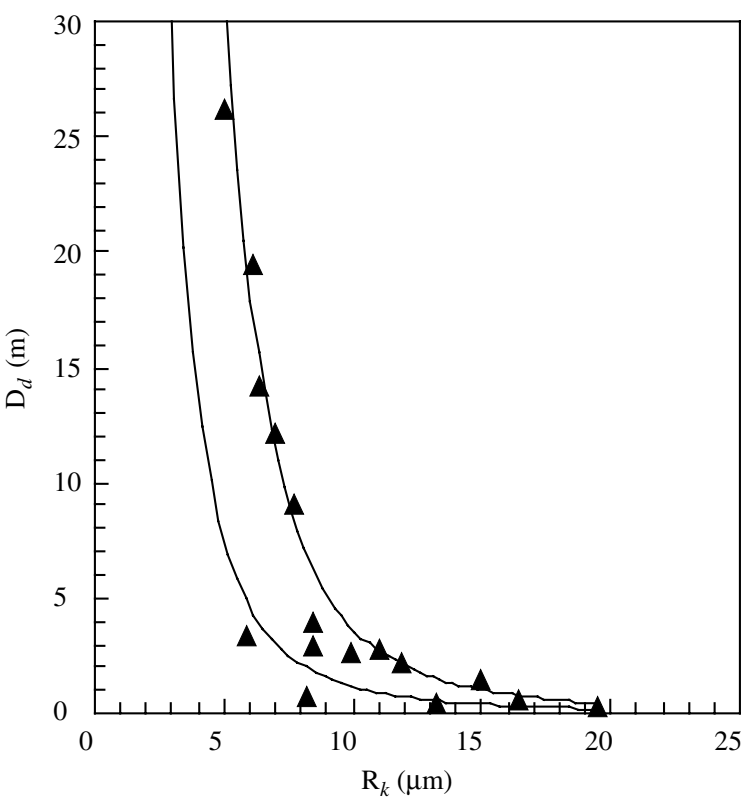

(b)

Figure 8. Effect of bearing ratio associated parameters on the durability distance: a) reduced peak height; b) core roughness depth.

\section{References}

1. Gopinath K. The influence of speed on the wear of sintered iron based materials. Wear. 1981; 71: 161-178.

2. Molinari A, Straffelini G. Wear process in high-strength sintered alloys under dry rolling-sliding. Wear. 1994; 173: 121-128.

3. Amsallem C, Gaucher A, Guillot G. The unlubricated frictional behavior of sintered iron. Wear. 1973; 23: 97-112.

4. Eyre TS, Walker RK. Wear of sintered materials. Powder Metallurgy. 1976; 1: 22-30.

5. Lim SC, Brunton JH. The unlubricated wear of sintered iron. Wear. 1986; 113: 371-382.

6. Leheup ER, Zhang D, Moon JR. Low amplitude reciprocating wear of sintered iron. Wear. 1994; 176: 121-130.

7. Leheup ER, Zhang D, Moon JR. The effect of density on fretting wear of sintered iron. Wear. 1994; 176: 111-119.

8. Leheup ER, Zhang D, Moon JR. Fretting wear of sintered iron under low normal pressure. Wear. 1998; 221: 86-92.

9. Beiss P. Steam treatment of sintered parts. Powder Metallurgy. 1991; 34: 173-177.

10. Razavizadeh K, Davies BL. Influence of powder type and density on pore closure and surface hardness changes resulting from steam treatment of sintered iron. Powder Metallurgy. 1979; 22: 187-192.

11. Molinari A. Straffelini G. Quality control of steam treated sintered iron: Importance of oxide characterisation. Surface Engineering, 1998; 14: 331-333.

12. Binder R. Influência dos processos de ferroxidação nas propriedades do ferro sinterisado", [M. Sc. Dissertation]. Florianopólis: Universidade Federal de Santa Catarina; 1996.
13. Franklin P, Davies BL. The effects of stream oxidation on porosity of sintered iron. Powder Metallurgy. 1977; 20: 11-16.

14. Razavizadeh K, Davies BL. Combined effects of steam treatment and age hardening on mechanical properties of sintered $\mathrm{Fe}-\mathrm{Cu}$ alloys. Powder Metallurgy. 1982; 25(1): 11-16.

15. Voleník K, Volrábová H, Neid J, Seberíni M. Structure of oxidation products of sintered steel in superheated steam. Powder Metallurgy. 1978; 21: 149-154.

16. De Mello JDB, Klein A, Binder R, Hutchings IM. Effect of compaction pressure and powder grade on the microstructure and hardness of steam oxidised sintered iron. Powder Metallurgy. 2001; 44: 1-9.

17. Ferrari A., Veronesi V, Gazzanica GC. A study of the influence of steam treatment on the tribological behavior of steel specimens. Wear. 1981; 66: 195-207.

18. Razavizadeh K, Davies BL. The effects of steam treatment on the wear resistance of sintered iron and Fe-Cu alloys. Wear. 1981; 69: 355-367.

19. Straffelini G, Molinari A. Dry sliding behavior of steam treated sintered iron alloys. Wear. 1992; 159: 127-134.

20. Molinari A, Straffelini G. Surface durability of steam treated sintered iron alloys. Wear. 1995; 181-183: 334-341.

21. Molinari A, Straffelini G. Tribological behavior of steam treated ferrous parts. International Journal of Powder Metallurgy. 1997; 32: 55-62.

22. De Mello J D B, Hutchings I M. Effect of processing parameters on the surface durability of steam oxidised sintered iron. Wear.2001; 250:435448.

23. Wyko Corporation. Surface Profiles: Technical reference manual. Wyko Corporation; Tucson, AZ, USA; 1996. 